\title{
Hypoxic-ischemic retinal injury in rat pups
}

\author{
Hsiu-Mei Huang ${ }^{1}$, Chao-Ching Huang ${ }^{2}$, Pi-Lien Hung ${ }^{3}$, Ying-Chao Chang ${ }^{3}$
}

BACKGROUND: Visual loss associated with brain damage, especially hypoxic-ischemic (HI) encephalopathy, is the most common cause of visual impairment in children in developed countries. We hypothesized that $\mathrm{HI}$ insults can cause long-term damage in immature eyes.

METHODS: In postnatal day 7 rat pups, HI was induced by unilateral common carotid artery ligation followed by hypoxia. Retina damage was assessed by electroretinography (ERG) and cell counting. Neuronal injury and astrogliosis were evaluated by terminal deoxynucleotidyl transferase nick-end labeling, cleaved caspase 3, ED1, and glial fibrillary acidic protein immunostaining.

RESULTS: We observed rapid and persistently extensive injuries in the ganglia cell layer (GCL), inner plexiform layer, and inner nuclear layer (INL) in ipsilateral retinas after $\mathrm{HI}$ injury, corresponding to the marked alteration in ERG. HI insult caused prominent microglial and Múller cell activation in ipsilateral inner retinas. Neuronal death in the GCL and INL after HI injury was mainly apoptotic, involving caspase-dependent pathways.

CONCLUSION: Our study demonstrated the first evidence of $\mathrm{HI}$ retinal damage at both the pathological and functional level using the Vannucci model in neonatal rats. Because retinal damage is often associated with HI injury, it is important to demonstrate that a particular neuroprotective strategy effectively preserves the retina in addition to the brain.

$\mathbf{P}$ erinatal hypoxic-ischemic (HI) injury is the most common cause of neurological deficits in immature brains (1). With advances in perinatal care, survival rates for infants with multiple handicaps after $\mathrm{HI}$ insults are increasing. The prevalence of visual impairment in children with $\mathrm{HI}$ injury ranges from 66 to 94\%. The visual dysfunctions associated with HI brain injury include strabismus, gaze palsy, nystagmus, optic atrophy, and restriction of the visual field $(2,3)$, defective color vision $(4,5)$, and reduced grating acuity (6). Visual loss associated with brain damage is currently the most common cause of visual impairment in children in developed countries, placing a major burden on ophthalmological and educational services in these countries (2).

Most studies have emphasized "cerebral visual impairment" in neurologically damaged children. These findings were presumably attributed to trans-synaptic degeneration of optic axons as a result of bilateral occipital damage because the eyes are more resistant than the brain after HI injury (7). However, although impaired visual acuity is frequent among infants with magnetic resonance imaging evidence of cerebral damage, the severity cannot be predicted on brain imaging (4). Furthermore, retinal ischemia is a common clinical condition in adults, occurring in a variety of ocular pathologies, including ischemic optic neuropathy, retinal vessel occlusion, diabetic retinopathy, and glaucoma (7). Several retinal ischemia models, including high intraocular pressure, vascular occlusion, and intravitreal injection of NMDA (N-methyl-D-aspartate), have been well established in adult rodents. A common hallmark for these conditions is the progressive degeneration and the final neuronal loss in the ganglion cell layer (GCL) (8-13). However, it remains to be determined whether the developing eyes are vulnerable to $\mathrm{HI}$ damage at both pathological and functional levels. We hypothesized that HI insult in the immature eyes may play a significant role in the visual impairment of these children.

The Vannucci model is one of the most widely used experimental paradigms to induce $\mathrm{HI}$ injury in rat pups, resembling HI damage to the human neonatal brain. In rat pups, unilateral common carotid artery ligation followed by hypoxia produces selective damage in the hemisphere ipsilateral to the artery occlusion (14). The ophthalmic artery mainly supplies the inner retina and originates from the internal carotid artery. Therefore, unilateral common carotid ligation simultaneously obstructs blood flow in the ipsilateral retina (15). Although the neuronal damage in the brain has been well studied in this model, little is known about the consequences of HI retinal injury in rat pups. Furthermore, because visual deficits may confound the behavioral outcomes as measured by the Morris water maze and radial arm maze tasks (16), it is important to delineate the long-term sequelae of retina injury after $\mathrm{HI}$ in rat pups.

\section{RESULTS}

HI Caused Long-Term Retinal Functional Impairment in Rat Pups To address the alteration of functional development after HI, sequential scotopic electroretinography (ERG) was obtained on postnatal day (P)14, P21, P28, P35, P42, P49, and P60 (Figure 1a-i). The a-wave is associated with rod photoreceptor activity and the b-wave reflects the combined activity of

'Department of Ophthamology, Kaohsiung Chang Gung Memorial Hospital and Chang Gung University College of Medicine, Kaohsiung, Taiwan; ${ }^{2}$ Institute of Clinical Medicine and Department of Pediatrics, National Cheng Kung University College of Medicine, Tainan, Taiwan; ${ }^{3}$ Department of Pediatrics, Kaohsiung Chang Gung Memorial Hospital and Chang Gung University College of Medicine, Kaohsiung, Taiwan. Correspondence: Ying-Chao Chang (chao8725@ms16.hinet.net) 
depolarizing bipolar cells and Müller cells (15). In naive rats, there was a rapid and significant growth in both $a-$ and $b$-wave amplitude that reached the maximum level on $P>1$, as documented previously (17). HI injury in rat pups markedly altered the normal maturation process of the ERG signal. After HI, responses obtained from the ipsilateral eyes already demonstrated significantly decreased a- and b-waves as compared with the contralateral eyes on eye opening (P14). On P21, the maximum a-wave amplitude in the ipsilateral eyes was significantly decreased relative to that in the contralateral eyes. From P28 to P60, there were no significant differences in a-waves between the ipsilateral, contralateral retina, and naive groups (Figure $\mathbf{1 j}$ ). In contrast to the relative resistance of the a-wave to $\mathrm{HI}$ injury, the maturation of the b-waves in ipsilateral eyes was markedly delayed for $7 \mathrm{~d}$ (P28). Furthermore, the amplitude of the b-waves in the ipsilateral eyes remained significantly lower than that in the contralateral eyes at all the time periods examined (Figure 1k).

\section{HI Caused Long-Term Retinal Damage at Pathological Levels}

The long-term maturational alteration in retinal function after $\mathrm{HI}$ injury in rat pups was accompanied by changes in retinal structure. Ipsilateral retina showed prominent pyknotic cells and tissue vacuolation within the GCL and inner nuclear layer (INL) as early as $3 \mathrm{~h}$ post-HI (Figure $2 \mathrm{c}$ ), whereas the naive (Figure 2a) and contralateral retinas (Figure 2b) did not reveal such cytoarchitecture changes. A significant progression in cell loss was noted within the GCL and INL of the ipsilateral retina from P8 to P60 (Figure 2c-1). In naive rats, the thickness of the inner plexiform layer (IPL) and outer plexiform layer grew from P8 to P21, after which a gradual thinning occurred. The ganglion cells increased, but the INL and outer nuclear layer thickness decreased as the animals grew. In the HI rats, the ipsilateral retina had significant decreases of GCL cell numbers (Figure 2m) and IPL thickness (Figure 2n) from P8 to P60 as compared with the contralateral retina. The ipsilateral retina also showed significantly thinner INL (Figure 20) and outer plexiform layer (Figure 2p) than the contralateral retina on P14 to P60. There was no significant difference in the thickness of outer nuclear layer between the $\mathrm{HI}$ and naive groups (Figure 2q).

HI caused acute hypoxic stress in the inner retina of the rat pups. On P33, hematoxylin and eosin staining demonstrated extensive neuronal injury throughout the cortex, striatum, and hippocampus in the ipsilateral hemisphere after HI (Figure 3b). The degree of brain injury, as measured by the degree of cerebral hemispheric weight reduction, was not correlated with the reduction of inner retinal thickness (Figure 3c, $r^{2}=$ $0.0314, P=0.59$ ).

To assess the retinal hypoxia in HI insults, we used hypoxyprobe-1 (HP-1) immunostaining. There was no HP-1 immunoreactivity in the contralateral retina of $\mathrm{HI}$ rats. However, several HP-1(+) cells were found in the GCL of ipsilateral eyes (Figure 3d). These data suggested that in addition to the brain, the ipsilateral retina also underwent the hypoxic stress.
HI injury induced astrogliosis in immature retinas. Activated microglia have been described as a "sensor" for pathological events in the central nervous system (18). We found that there were very few ED1(+)cells in the GCL and IPL in the naive and contralateral retina (Figure 4a,b). At $6 \mathrm{~h}$ after HI, there were many microglia cells in the amoeboid form. The number of ED1(+) cells increased gradually in the GCL and the IPL from $6 \mathrm{~h}$ post-HI, reached a peak level on $\mathrm{P} 9$, and decreased from P14 (Figure 4c-l).

During neuronal damage, Múller cells undergo reactive gliosis characterized by the upregulation of intermediate filaments such as glial fibrillary acidic protein (GFAP) (19). The GFAP immunoreactivity was restricted to the astrocytes of the GCL in both the HI and naive rats initially (Figure $4 \mathrm{~m}-\mathrm{q}$ ). As compared with the contralateral retina, there were prominent increases of GFAP immunoreactivity in the Müller cell trunks throughout the whole ipsilateral retina layers from P21 to P60 after HI (Figure $4 \mathbf{r}-\mathbf{t}$ ).

\section{HI Retinal Injury Involved Apoptotic Neuronal Death}

Terminal deoxynucleotidyl transferase nick-end labeling (TUNEL) staining showed a gradual increase in TUNEL $(+)$ cells in the ipsilateral GCL and INL from $6 \mathrm{~h}$ post-HI, a peak ay $24 \mathrm{~h}$ post-HI, and then a decrease after P9 (Figure $5 \mathrm{c}-\mathbf{j}$ ). In the HI group, the ipsilateral retina had a significant increase in the number of TUNEL $(+)$ cells $\left(22.86 \pm 14.62 / 4000 \mu \mathrm{m}^{2}\right)$ relative to the contralateral retina $(3.91 \pm 2.23)$ and the naive group $(4.16 \pm 1.45) 24 \mathrm{~h}$ post-HI $(P<0.0005)$. The numbers of cleaved caspase-3(+) cells in the GCL and INL of the ipsilateral retinas also increased from $3 \mathrm{~h}$ post-HI and reached a peak on P8 (Figure $5 \mathrm{~m}-\mathbf{q}$ ).

\section{DISCUSSION}

To the best of our knowledge, this is the first study to demonstrate evidence of HI retinal damage at both the pathological and functional level using the Vannucci model in neonatal rats. The injuries were rapid ( $3 \mathrm{~h}$ after $\mathrm{HI}$ ) and extensive in GCL $(60 \%$ cell loss), IPL (55\% thickness reduction), and INL ( $45 \%$ cell loss). The pathological evidence of retinal injury corresponded to the marked suppression of the amplitude of b-waves (60\%) in ERG, even on P60. HI insults in neonatal rats caused severe hypoxic stress in ganglia cells, prominent astrogliotic response, and apoptotic neuronal death in the inner retina.

These findings are in contrast in both temporal and spatial aspects to the previous studies that provided evidence of retinal ischemia in adult rodents. Permanent bilateral middle cerebral artery ligation in adult rats produced only subtle retinal changes (20). Bilateral common carotid occlusion induced damage in the GCL and INL at $1 \mathrm{wk}$ and 2 mo, respectively, after injury in adult Wistar rats (13). Transient middle cerebral artery occlusion resulted in pyknotic cells in the GCL and INL $2 \mathrm{~h}$ after reperfusion. However, no loss of cells within the ischemic retina was observed $1 \mathrm{~d}$ after reperfusion in adult SV129EV mice (15). Central retinal artery occlusion significantly reduced GCL cell counts and retinal thickness at $21 \mathrm{~d}$ after ischemic injury in adult mice (11). In our study, there were many pyknotic cells 


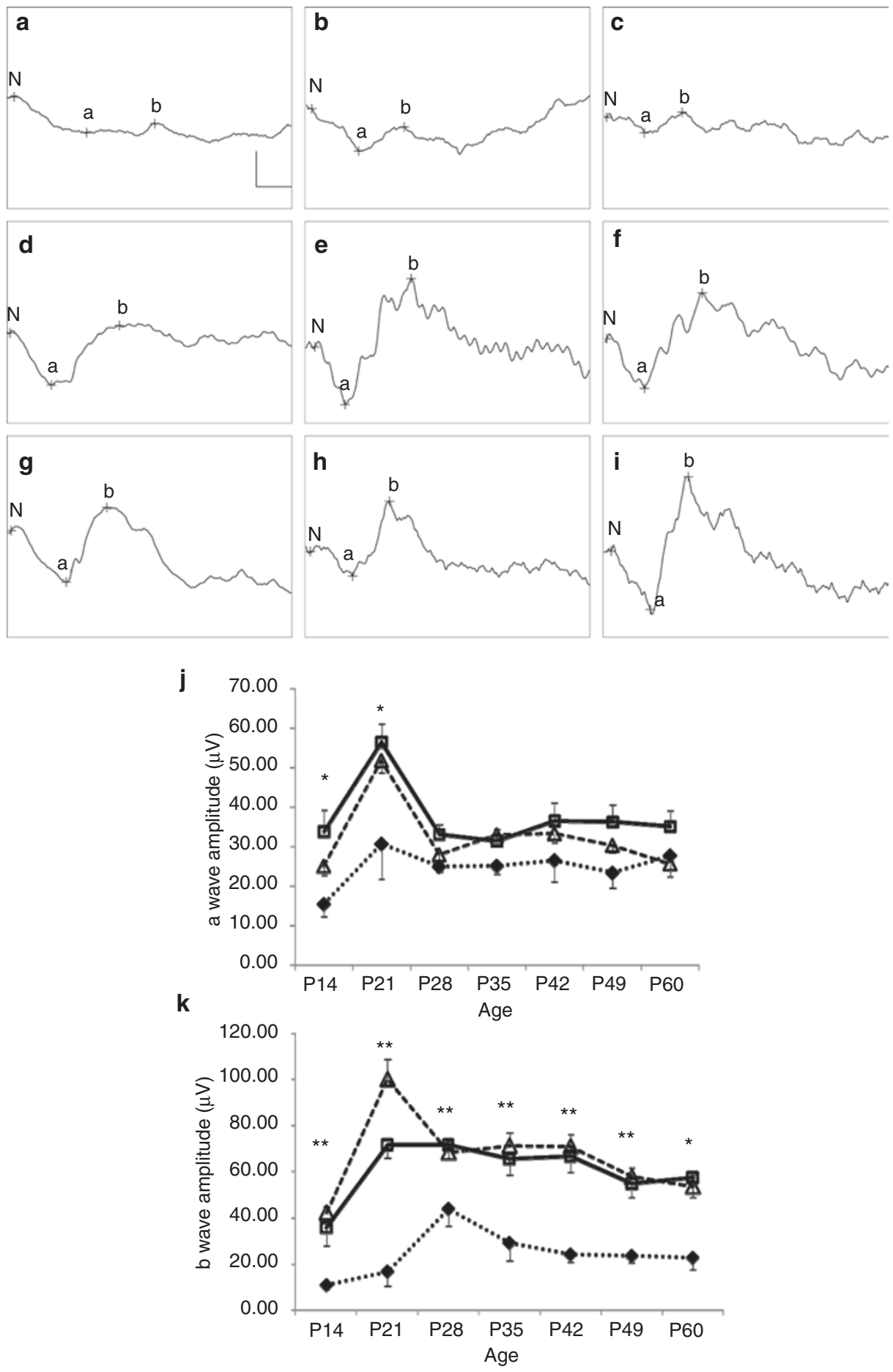

Figure 1. Hypoxia-ischemia (HI) causes long-term alterations of retinal function maturation in rat pups. Representative electroretinogram (ERG) obtained from the (a-c) ipsilateral and (d- $\mathbf{f})$ contralateral eyes of $\mathrm{HI}$ and $(\mathbf{g}-\mathbf{i})$ naive rats on $(\mathbf{a}, \mathbf{d}, \mathbf{g})$ postnatal day (P)21, (b,e,h) P33, and (c,f,i) P60. Group data of ERG obtained on P15, P21, P28, P35, P42, P49, and P60 shows that there was a rapid growth in (j) a- and (k) b-wave amplitude to a maximum reached on P21 in naive rats (open triangles). Significantly decreased a-wave amplitude from ipsilateral eyes of $\mathrm{HI}$ rats (filled diamonds) was noted from P14 to P21. The maturation of the b-waves in ipsilateral eyes was markedly halted and delayed for $7 \mathrm{~d}$ (P28). Furthermore, the amplitude of the ipsilateral b-waves remained significantly lower as compared with those of the contralateral eyes (open squares) of $\mathrm{HI}$ rats in all the time periods examined. Calibration: horizontal, $20 \mathrm{~ms}$; vertical $50 \mu \mathrm{V}$. N, baseline; a, trough of a-wave; b, peak of b wave. ${ }^{*} P<0.05 ;{ }^{*} P<0.01$ vs. ipsilateral retina of $\mathrm{HI}$ rats. $n=5-10$ for each time point.

and there was tissue vacuolation within the GCL and INL in the ipsilateral retina $3 \mathrm{~h}$ after HI. Significant cell loss in the GCL and thickness reduction in the IPL was observed as early as $1 \mathrm{~d}$ after HI. Furthermore, there was prominent reduction of thickness in the outer retina (outer plexiform layer), in addition to the inner retina (GCL, IPL, and INL) in the ipsilateral retina from P14 to P33. This suggested that immature retinas are more susceptible to $\mathrm{HI}$ injury as compared with those of mature rats. 

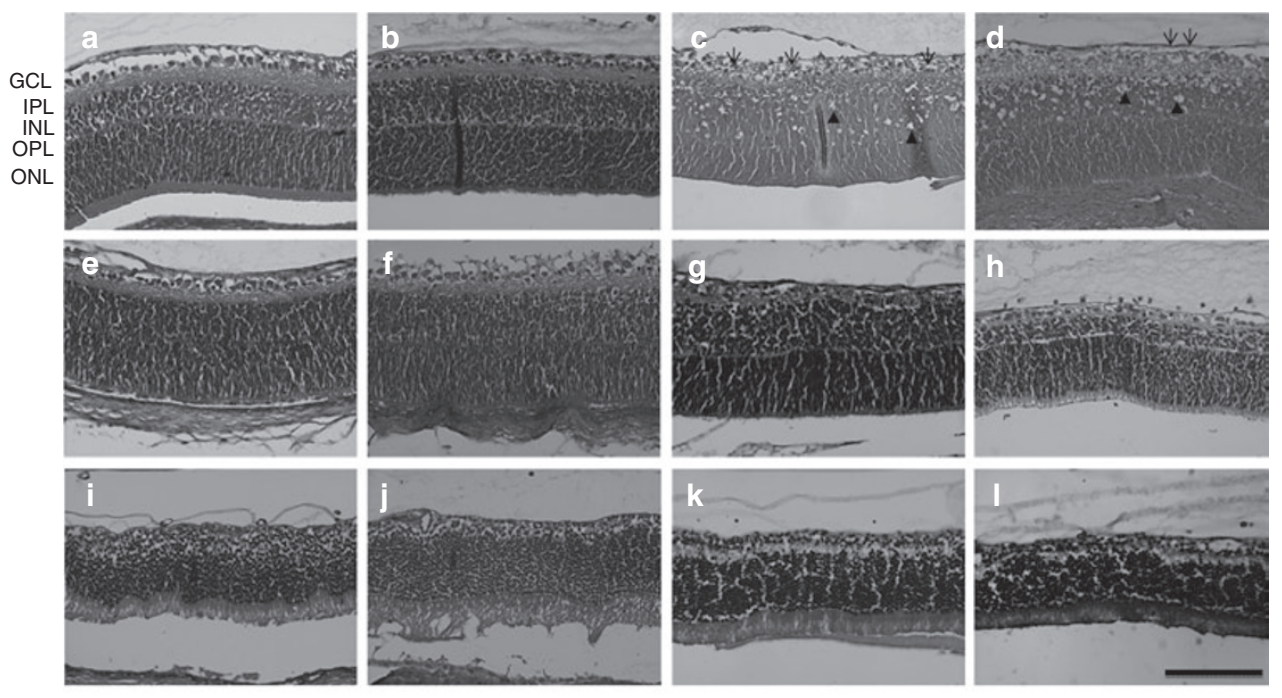

m
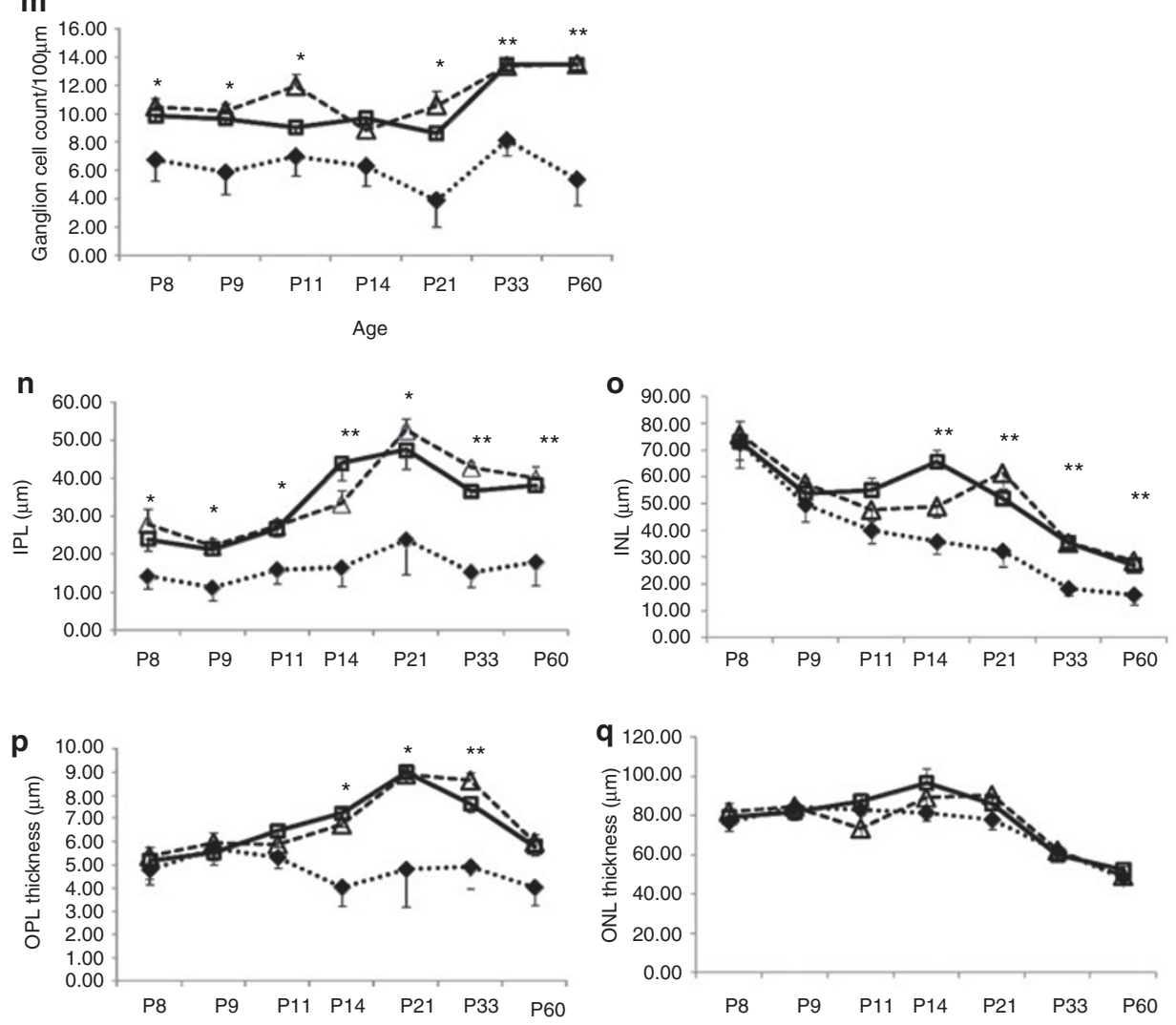

Figure 2. Hypoxia-ischemia $(\mathrm{HI})$ causes long-term retinal damage in rat pups. Representative retinal histological sections from (a) naive retina, (b) contralateral retina, and (c-I) ipsilateral retina shows prominent pyknotic cells (arrows) and tissue vacuolation (arrowheads) within the ganglion cell layer (GCL) and inner nuclear layer (INL) (c) $3 \mathrm{~h}$ and (d) $6 \mathrm{~h}$ post-HI. There was progressive cell loss from (e) $12 \mathrm{~h}$ and (f) $18 \mathrm{~h}$ post-HI, (g) P8, (h) P9, (i) P14, (j) P21, (k) P33 to (I) P60 within the GCL and INL of the ipsilateral retina. Group data showed that the HI rats had significantly decreased (m) GCL cell numbers and (n) inner plexiform layer (IPL) thickness in the ipsilateral retina (filled diamonds) from P8 to P60, as compared with the contralateral retina (open squares) of $\mathrm{HI}$ rats and the naive group (open triangles). The ipsilateral retina of the HI rats also showed a thinner (o) INL and (p) outer plexiform layer than the contralateral retina and those of naive rats, which reached significance on P14 to P60. (q) There was no significant difference in the thickness of outer nuclear layer between the $\mathrm{HI}$ and naive groups. ${ }^{*} P<0.05 ;{ }^{* *} P<0.01$ vs. ipsilateral retina of $\mathrm{HI}$ rats. Arrows: pyknotic cells in the GCL. Arrowheads: tissue vacuolation in the INL. Bar: $100 \mu \mathrm{m} . n=6-10$ for each time point.

In this study, microglial activation appears early ( $6 \mathrm{~h}$ post-HI) and subsides in the recovery phase (P14), suggesting a possible early role in the pathophysiology of immature retinal HI injury. The selective increases of ED1 immunoreactivities in the inner retina also corresponded to the region-specific vulnerability of immature retina to $\mathrm{HI}$ injury. Activated microglia are the hallmark of neuroinflammation and exacerbate cerebral neuronal injury through production of proinflammatory cytokines (21). 
a

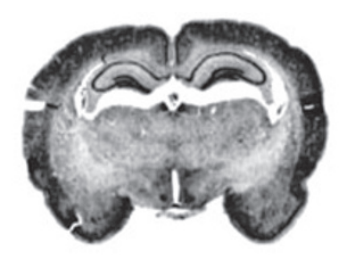

b

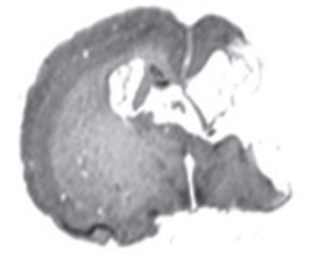

d

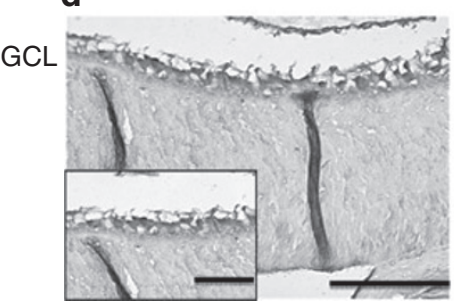

C

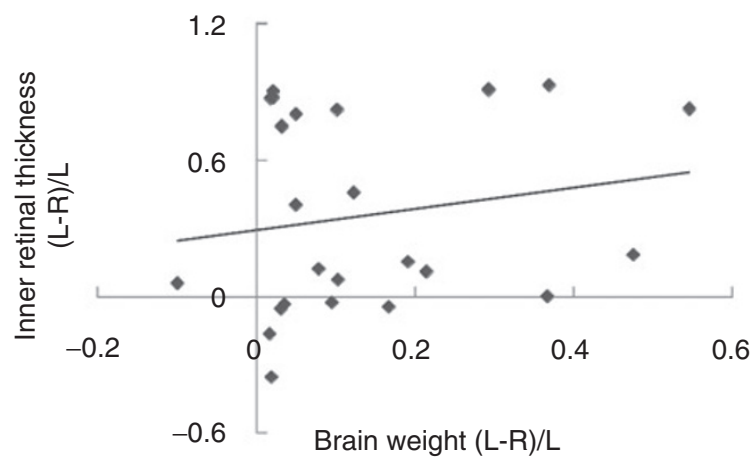

f

e

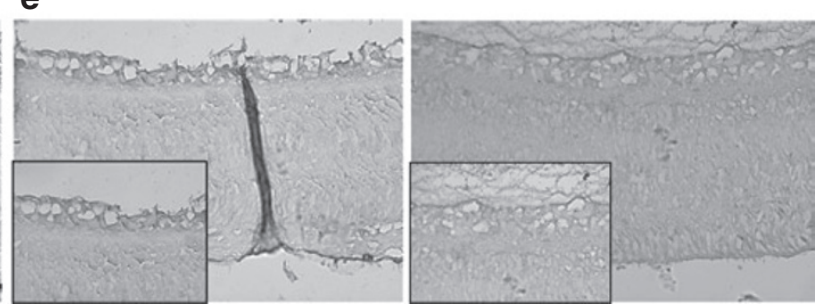

Figure 3. Hypoxia-ischemia (HI) caused hypoxic stress in the immature retina. Cresyl violet staining of brain sections demonstrated extensive neuronal injury throughout the (b) ipsilateral cortex, striatum, and hippocampus after $\mathrm{HI}$ injury in neonatal rats, as compared with the (a) naive rat brain. (c) There was no significant correlation between the reduction of cerebral hemispheric weight and the inner retinal thickness in $\mathrm{HI}$ rats on P33 $\left(r^{2}=0.0314, P=0.59\right.$, $n=20$ ). (d) Representative Hypoxyprobe-1 (HP-1) immunostaining of the retinas demonstrated several HP(+) cells located in the ganglion cell layer (GCL) of the ipsilateral eyes. In contrast, there were no HP-1(+) cells in the (e) contralateral eyes of HI rats or (f) those of naive rats. L, left side; R, right side; Bar, $100 \mu \mathrm{m} ; 50 \mu \mathrm{m}$ in inset. $n=3$ for each group.

Microglial activation has also been demonstrated in various pathologic conditions of the retina, such as glaucoma, diabetic retinopathy, and axotomized retina in adult rodents $(18,22)$. Our data show that microglia activation is also important in early $\mathrm{HI}$ retinal injury in neonatal rats. In contrast to the early microglial activation, we did not found prominent GFAP immuoreactivity until P21. However, very intense GFAP labeling was seen by $1 \mathrm{wk}$ after retinal ischemia in adult rats (13). During retinal injury, Müller cells are well known to undergo reactive gliosis characterized by the upregulation of GFAP (19). Müller cells, the main type of glial cell in the retina, are responsible for the maintenance of homeostasis in the extracellular medium of the retina, and protect the neurons by releasing neurotrophic factors and secreting glutathione, which has an antioxidant effect (23). The relative delayed reaction of Müller cells, as compared with those of the adult rats, may contribute to the increased vulnerability of immature retina to $\mathrm{HI}$ injury. An improved understanding of the different gliotic response and of their protective or damaging effects is essential for the development of effective treatment strategies for HI retinal injury in rat pups.

The increased vulnerability of immature retinas to $\mathrm{HI}$ injury may also be related to the prominent apoptotic cell death. TUNEL has been shown to be rarely detectable in ischemic retinas up to $1 \mathrm{~d}$ after reperfusion in adult mice (15). In contrast, there was a significantly increased number of TUNEL $(+)$ cells in ipsilateral retinas as early as $6 \mathrm{~h}$ post-HI in rat pups. Furthermore, the prominent apoptosis in immature retinas was related to activation of caspase-dependent pathways, as demonstrated by caspase- 3 cleavage. It remained to be determined whether prevention of caspase activation is protective in neonatal retinal injury, similar to previous reports on $\mathrm{HI}$ brain injury (24).

Previous studies have emphasized that the eyes were more resistant than the brain after HI injury (7). The visual impairments in the brain-damaged infants were attributed to occipital damage. However, our data show that HI insult in neonatal rats can cause significant hypoxic stress, apoptosis, and neuroinflammation in the immature retina. There was also no significant correlation between the cerebral hemispheric weight and inner retinal thickness reduction, which related to the infarct brain area and retinal injury, respectively. Furthermore, if the retinal injury is due to trans-synaptic degeneration of brain insult, the left retina should show more severe injury than the right side does. The clinical studies have observed that the pattern and severity of visual deficits cannot be predicted on brain magnetic resonance imagings in infants (4). Our study provides the evidence that retinal injury contributes significantly to the visual impairments in neonates with HI brain injury.

The Vannucci model is one of the most widely used experimental paradigms to induce $\mathrm{HI}$ injury in rat pups, and resembles HI damage to the human neonatal brain. However, little is known about the consequences of $\mathrm{HI}$ injury in immature retinas. Furthermore, because visual deficits may confound the behavioral outcomes as measured by the Morris water maze (15), it is 

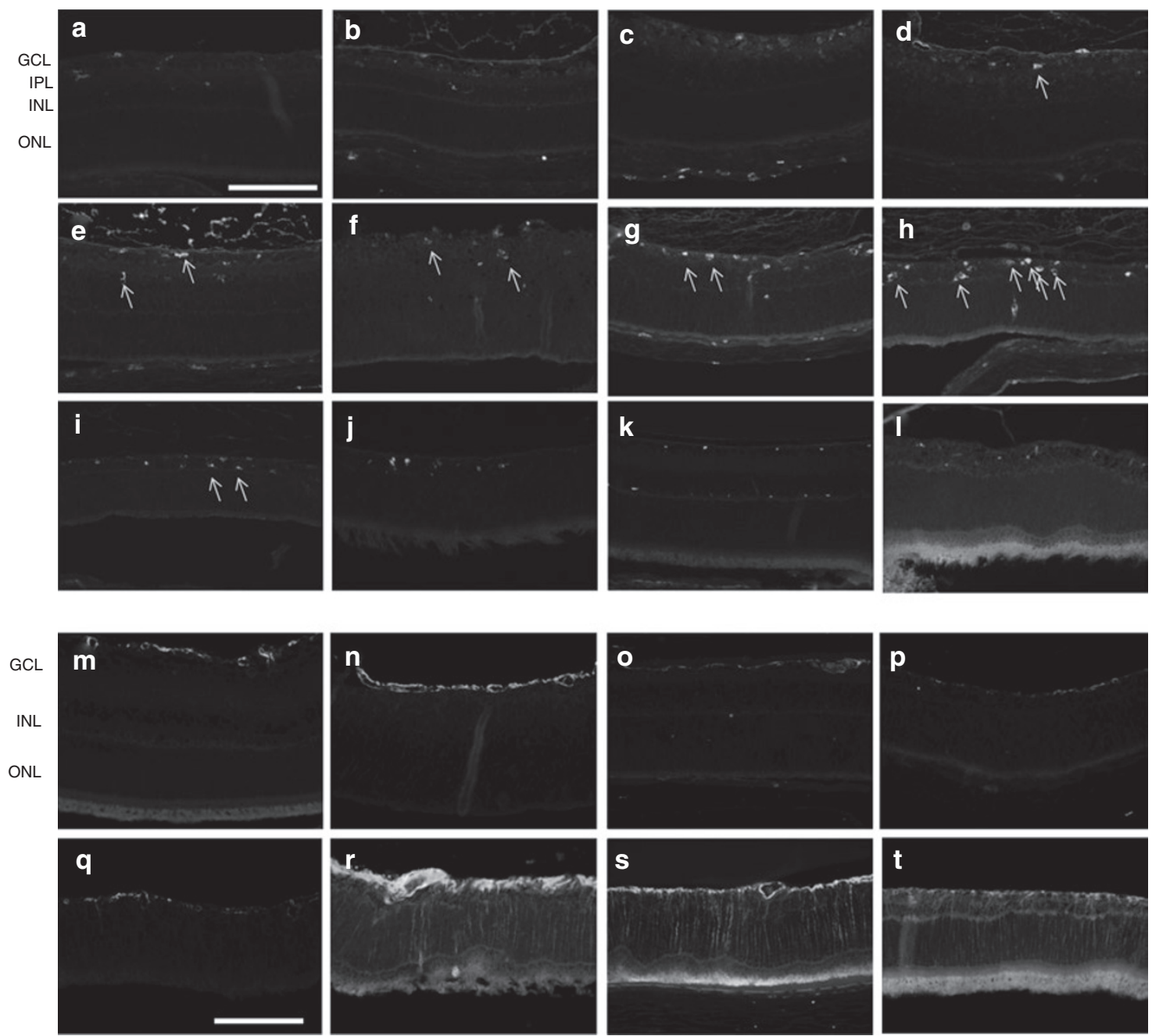

Figure 4. Hypoxia-ischemia (HI) causes astrogliotic response in the immature retina. ED1 immunostaining showed that there were very few ED1(+) cells in the (a) naive, (b) contralateral, and (c) ipsilateral retina $3 \mathrm{~h}$ post-HI. The number of ED1(+) cells, representing activated microglia, increased gradually in the ganglion cell layer (GCL) and the inner plexiform layer (IPL) from (d) $6 \mathrm{~h},(\mathbf{e}) 12 \mathrm{~h}$, and (f) $18 \mathrm{~h}$ post-HI, (g) P8 to (h) P9 in the ipsilateral retina of HI rats, after which a gradual decrease occurred on (i) P11, (j) P14, (k) P21, and (I) P33. GFAP immunoreactivity was restricted to the astrocytes of the GCL in (m) contralateral and ipsilateral retinas of $\mathrm{HI}$ rats on (n) P8, (o) P9, (p) P11, and (q) P14. There was prominently increased GFAP immunoreactivity throughout the whole ipsilateral retina layers on (r) P21, (s) P33, and (t) P60. Arrows: ED1(+) cells. Bar: $100 \mu \mathrm{m} . n=3-5$ for each group. INL, inner nuclear layer; ONL, outer nuclear layer.

important to delineate the effects of HI on vision. Our data show the long-term sequelae of HI retinal injury in neonatal rats, at both pathological and functional levels. As retinal ischemia is often associated with neonatal HI injury, it is important to demonstrate that a particular neuroprotective strategy effectively preserves the retina in addition to the brain.

\section{METHODS}

This study was approved by the Chang Gung Memorial Hospital Committee on Use and Care of Animals. Ten to 12 Sprague-Dawley pups per dam were used and housed with a 12/12-h light/dark schedule in a temperature- and humidity-controlled colony room. The pups were housed with their dams until weaning on P21, and then housed in groups of four to five per cage.

\section{HI Injury Model}

On P7, animals were anesthetized with $2.5 \%$ halothane (balance, room air), and the right common carotid artery was surgically exposed and permanently ligated with 5-0 surgical silk. After surgery, the pups were returned to the dam for a $1 \mathrm{~h}$ recuperation period before HI. The animals were then placed in airtight $500 \mathrm{ml}$ containers partially submerged in a $37^{\circ} \mathrm{C}$ water bath through which humidified $3 \mathrm{l} / \mathrm{min}$ $8 \%$ oxygen (balance, nitrogen) was maintained for $2 \mathrm{~h}$ (25). After completion of $\mathrm{HI}$, the rat pups were returned to their cages. The naive group did not receive any procedure.

\section{Long-Term Outcome Measures}

Hemispheric brain weight reduction. On P33, following deep anesthesia with an overdose of chloral hydrate $500 \mathrm{mg} / \mathrm{kg}$ i.p., rats were killed. The brains and eyes were removed, weighted, fixed, and then embedded in paraffin as described previously (26). After removal of the brainstem and cerebellum, the forebrain was sectioned at the midline, and left and right hemispheric weights were determined. The percentage of hemispheric weight reduction measured as (left hemisphere weight - right hemisphere weight)/left hemisphere weight was used as the measure of cerebral injury in this study. The changes in the hemispheric weight reduction were highly correlated to the changes in the infarct brain areas and the hemispheric volume changes (27).

Assessment of retinal injury. The eyes were immersed in $4 \%$ paraformaldehyde, dehydrated through graded alcohols, embedded in paraffin, and then coronally sectioned ( $8 \mu \mathrm{m}$ thick). Two sections per retina were randomly selected for hematoxylin and eosin staining. Images were 

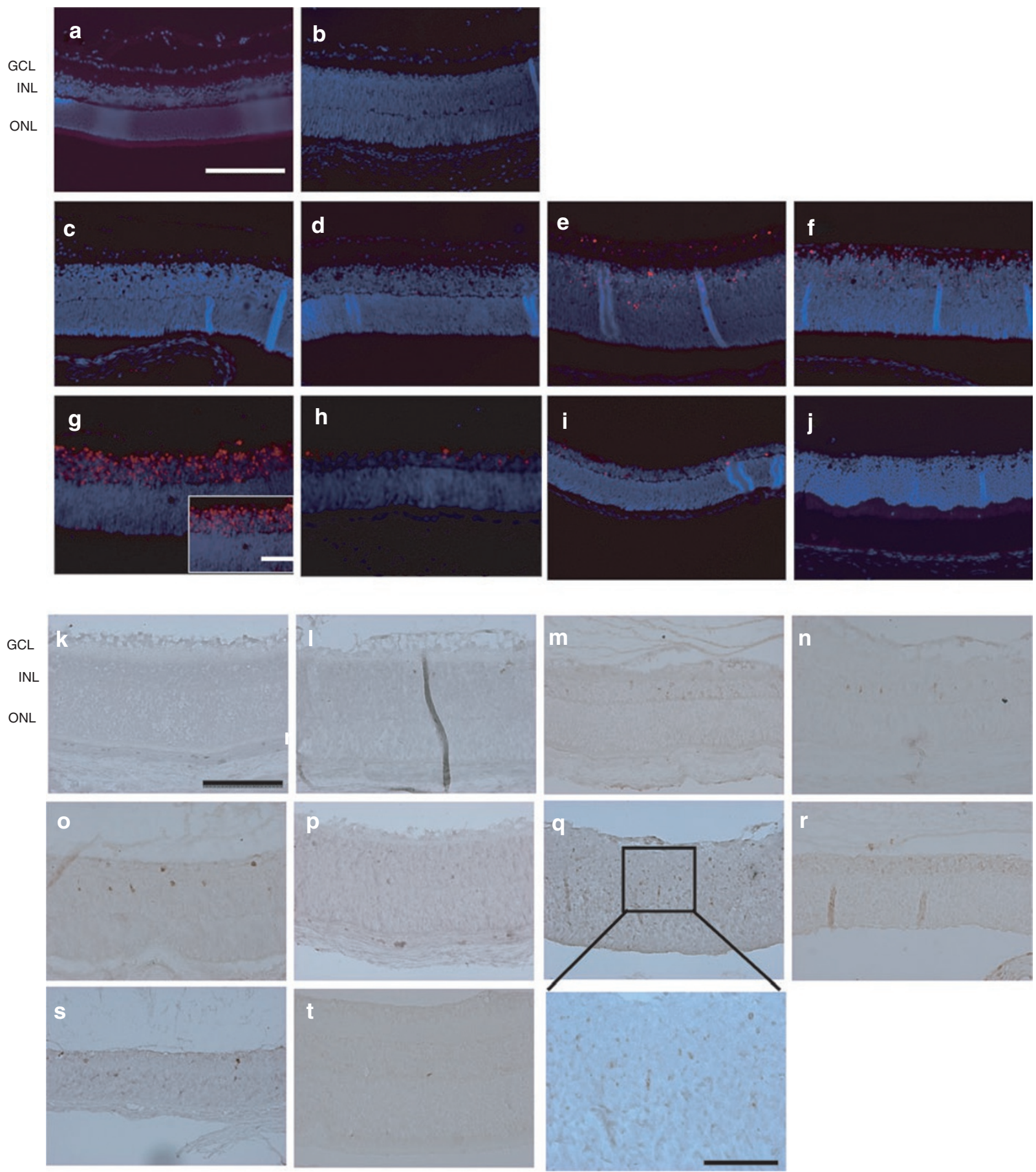

Figure 5. Hypoxia-ischemia (HI) caused apoptotic cell death in immature inner retina. TUNEL staining showed very little immunodensity in the (a) naive, (b) contralateral, and (c) ipsilateral retinas $3 \mathrm{~h}$ post-HI. A gradual increase of TUNEL(+) cells in the ipsilateral ganglion cell layer (GCL) and the inner nuclear layer (INL) from (d) $6 \mathrm{~h},(\mathbf{e}) 12 \mathrm{~h}$ to (f) $18 \mathrm{~h}$ post-HI, which peaked on (g) P8, and then decreased from (h) P9, (i) P11 to (j) P14. There was a gradual increase in cleaved caspase-3(+) cells in the GCL and the INL of the ipsilateral retina from $(\mathbf{m}) 3 \mathrm{~h},(\mathbf{n}) 6 \mathrm{~h},(\mathbf{o}) 12 \mathrm{~h}(\mathrm{O})$, and (p) $18 \mathrm{~h}$ post-HI to (q) P8 (inset below) in the $\mathrm{HI}$ rats, as compared with the (I) contralateral retina and the (k) naive group. The cleaved caspase-3 immunodensity in the ipsilateral retina then decreased from (r) P9, (s) P11 to (t) P14. Bar: $100 \mu \mathrm{m} ; 50 \mu \mathrm{m}$ in inset. $n=6$ for each group. ONL, outer nuclear layer; TUNEL, terminal deoxynucleotidyl transferase-mediated dUTP nick-end labeling.

acquired using a Nikon microscope (Nikon, Tokyo, Japan) (25). To quantify the extent of retinal damage, cells were counted in two nonoverlapping views $(250 \times 250 \mu \mathrm{m})$ within central areas $(100 \mu \mathrm{m}$ from the optic disc) from each section by two independent observers (15).

Functional evaluation by ERG. The pupils of rats were dilated by $1 \%$ Mydriacyl (Alcon, Puurs, Belgium) and 1\% Cyclogyl (Alcon), then dark-adapted for $1 \mathrm{~h}$ before recording flash ERG (UTAS-E 300; LKC Technology, Gaithersburg, MD). After the rats had been anesthetized by $10 \%$ atropine i.m. and $6 \%$ choloral hydrate i.p., the recording electrode was placed on the cornea with $0.5 \%$ methyl cellulose as a conductive medium. A reference electrode was inserted in the subcutaneous area of the forehead, and a ground electrode was inserted in subcutaneous tissue on the back. The responses to a single-flash 
light (duration, $100 \mathrm{~ms}$ ) were amplified with a gain setting $\pm 500 \mu \mathrm{V}$ (filter: low, $0.3 \mathrm{~Hz}$; high, $500 \mathrm{~Hz}$ ). The amplitudes and the implicit times of the a- and b-waves were measured and averaged.

\section{Immunohistochemical Staining}

Paraffin sections were dewaxed, hydrated through graded alcohols, and placed in phosphate buffered saline. After being blocked by $5 \%$ goat serum, the sections were incubated with various primary antibodies overnight followed by secondary antibodies (Santa Cruz Biotechnology, Santa Cruz, CA) (25). The primary antibodies used were glial fibrillary acidic protein (1:200, GFAP, Chemicon, Temecula, CA), ED1 (1: 500, Serotec, Raleigh, NC), and activated caspase-3 (1:200, Cell Signaling, Danvers, MA). The sections were developed in 3'3-diaminobenzidine (Sigma Chemical, St Louis, MO) with a Vectastatin ABC system (Vector Laboratories, Burlingame, CA).

\section{Detection of Hypoxic Cells With Hypoxyprobe-1}

To confirm the hypoxic state of the retina during HI, the retina was immunostained with Hypoxyprobe-1 (HP-1, Chemicon, Burlington, MA), known as pimonidazole hydrochloride, which forms irreversible adducts with intracellular and extracellular proteins under hypoxic conditions ( $\mathrm{PO}<10 \mathrm{~mm} \mathrm{Hg}$ ). One hour after $\mathrm{HI}$, pups received $60 \mathrm{mg} /$ $\mathrm{kg} \mathrm{HP}-1$ i.p. and then were killed $2 \mathrm{~h}$ later. Pimonidazole adducts were detected in $4-\mu \mathrm{m}$ paraffin retinal sections using biotin-streptavidinperoxidase immunostaining.

\section{TUNEL}

TUNEL was performed using an ApopTag fluorescent direct in situ apoptosis detection kit (Chemicon), and samples were counterstained with Hoechst 33258 (Invitrogen, Carlsbad, CA) (25). The number of TUNEL(+) cells was counted in an area of $40 \times 100 \mu \mathrm{m}$ at $200 \times$ magnification, using a fluorescence microscope (Nikon).

\section{Statistical Analysis}

Statistical analyses were performed using GraphPad Prism 4 software (GraphPad Software, San Diego, CA). Statistical comparisons were performed using one-way ANOVA or two-tailed Student's $t$-tests. Values were considered significant at $P<0.05$, and data were presented as means \pm SEM.

\section{STATEMENT OF FINANCIAL SUPPORT}

This study was supported by grants CMRPG866193 and CMRPG880801 from Kaohsiung Chang Gung Memorial Hospital and Chang Gung University College of Medicine.

\section{REFERENCES}

1. Ferriero DM. Neonatal brain injury. N Engl J Med 2004;351:1985-95.

2. Hoyt CS. Brain injury and the eye. Eye (Lond) 2007;21:1285-9.

3. van Hof-Van Duin J, Evenhuis-van Leunen A, Mohn G, Baerts W, Fetter WP. Effects of very low birth weight (VLBW) on visual development during the first year after term. Early Hum Dev 1989;20:255-66.

4. Pike MG, Holmstrom G, de Vries LS, et al. Patterns of visual impairment associated with lesions of the preterm infant brain. Dev Med Child Neurol 1994;36:849-62.

5. Jacobson L, Ek V, Fernell E, Flodmark O, Broberger U. Visual impairment in preterm children with periventricular leukomalacia-visual, cognitive and neuropaediatric characteristics related to cerebral imaging. Dev Med Child Neurol 1996;38:724-35.

6. Luna B, Dobson V, Scher MS, Guthrie RD. Grating acuity and visual field development in infants following perinatal asphyxia. Dev Med Child Neurol 1995;37:330-44.

7. Osborne NN, Casson RJ, Wood JP, Chidlow G, Graham M, Melena J. Retinal ischemia: mechanisms of damage and potential therapeutic strategies. Prog Retin Eye Res 2004;23:91-147.
8. Takamatsu J, Hirano A, Levy D, Henkind P. Experimental bilateral carotid artery occlusion: a study of the optic nerve in the rat. Neuropathol Appl Neurobiol 1984;10:423-8.

9. Stevens WD, Fortin T, Pappas BA. Retinal and optic nerve degeneration after chronic carotid ligation: time course and role of light exposure. Stroke 2002;33:1107-12.

10. Wakita $\mathrm{H}$, Tomimoto $\mathrm{H}$, Akiguchi I, et al. Axonal damage and demyelination in the white matter after chronic cerebral hypoperfusion in the rat. Brain Res 2002;924:63-70.

11. Goldenberg-Cohen N, Dadon S, Avraham BC, et al. Molecular and histological changes following central retinal artery occlusion in a mouse model. Exp Eye Res 2008;87:327-33.

12. Ito $\mathrm{Y}$, Shimazawa M, Inokuchi $\mathrm{Y}$, et al. Degenerative alterations in the visual pathway after NMDA-induced retinal damage in mice. Brain Res 2008;1212:89-101.

13. Yamamoto H, Schmidt-Kastner R, Hamasaki DI, Yamamoto H, Parel JM. Complex neurodegeneration in retina following moderate ischemia induced by bilateral common carotid artery occlusion in Wistar rats. Exp Eye Res 2006;82:767-79.

14. Vannucci SJ, Hagberg H. Hypoxia-ischemia in the immature brain. J Exp Biol 2004;207(Pt 18):3149-54.

15. Steele EC Jr, Guo Q, Namura S. Filamentous middle cerebral artery occlusion causes ischemic damage to the retina in mice. Stroke 2008;39:2099-104.

16. Spencer RL, O'Steen WK, McEwen BS. Water maze performance of aged Sprague-Dawley rats in relation to retinal morphologic measures. Behav Brain Res 1995;68:139-50.

17. Dorfman A, Dembinska O, Chemtob S, Lachapelle P. Early manifestations of postnatal hyperoxia on the retinal structure and function of the neonatal rat. Invest Ophthalmol Vis Sci 2008;49:458-66.

18. Zhang C, Lam TT, Tso MO. Heterogeneous populations of microglia/macrophages in the retina and their activation after retinal ischemia and reperfusion injury. Exp Eye Res 2005;81:700-9.

19. Hirrlinger PG, Ulbricht E, Iandiev I, Reichenbach A, Pannicke T. Alterations in protein expression and membrane properties during Müller cell gliosis in a murine model of transient retinal ischemia. Neurosci Lett 2010;472:73-8.

20. Barnett NL, Osborne NN. Prolonged bilateral carotid artery occlusion induces electrophysiological and immunohistochemical changes to the rat retina without causing histological damage. Exp Eye Res 1995;61:83-90.

21. Gao HM, Hong JS. Why neurodegenerative diseases are progressive: uncontrolled inflammation drives disease progression. Trends Immunol 2008;29:357-65.

22. Zhang C, Tso MO. Characterization of activated retinal microglia following optic axotomy. J Neurosci Res 2003;73:840-5.

23. Vidal L, Díaz F, Villena A, Moreno M, Campos JG, Pérez de Vargas I. Reaction of Müller cells in an experimental rat model of increased intraocular pressure following timolol, latanoprost and brimonidine. Brain Res Bull 2010;82:18-24.

24. Zhu C, Wang X, Huang Z, et al. Apoptosis-inducing factor is a major contributor to neuronal loss induced by neonatal cerebral hypoxia-ischemia. Cell Death Differ 2007;14:775-84.

25. Lin WY, Chang YC, Lee HT, Huang CC. CREB activation in the rapid, intermediate, and delayed ischemic preconditioning against hypoxic-ischemia in neonatal rat. J Neurochem 2009;108:847-59.

26. Chang YC, Huang AM, Kuo YM, Wang ST, Chang YY, Huang CC. Febrile seizures impair memory and cAMP response-element binding protein activation. Ann Neurol 2003;54:706-18.

27. Lee HT, Chang YC, Wang LY, Wang ST, Huang CC, Ho CJ. cAMP response element-binding protein activation in ligation preconditioning in neonatal brain. Ann Neurol 2004;56:611-23. 